\title{
Effects of acellular dermal matrix on the prevention of complications and aesthetic outcome after parotidectomy
}

\author{
Jian-hui $\mathrm{Wu}^{1}$, Wei Zeng ${ }^{1}, \mathrm{Min}^{-Y i} \mathrm{Fu}^{1}$, and Fei Ye${ }^{1}$ \\ ${ }^{1}$ Sun Yat-Sen University
}

October 1, 2020

\begin{abstract}
Objective: The present study was designed to investigate whether acellular dermal matrix (ADM) graft could prevent Frey's syndrome (FS) and improve aesthetic score following parotidectomy. Study Design: Retrospective study. A propensity score matched analysis. Setting:From January 2015 to December 2019, 175 patients underwent parotidectomy. Methods: We divided the patients into two groups with ADM group and Control group. We included each group of 30 patients according a propensity score matched analysis. Results: The subjective of FS was recorded in 1 patient (3\%) from the ADM group and 9 patients (30\%) from the Control group $(\mathrm{P}=0.015)$. Patients in the ADM group had a subjective aesthetic score of $6.1+1.7$ compared with $5.2+1.7$ in the Control group. The subjective aesthetic score for patients in the ADM group was higher than those in the Control group $(\mathrm{P}=0.040)$. Conclusion: The present clinical study suggests that ADM graft are effective in preventing FS and improving aesthetic score after parotidectomy.
\end{abstract}

\section{INTRODUCTION}

The approximately $80 \%$ of parotid tumors are benign tumor, and surgical removal is the primary treatment for parotid tumors. (1) Benign tumors are commonly located in the superficial lobe. Complete excision of benign parotid tumors with an adequate margin of normal tissue is recommended, resulting in partial superficial, superficial, or even subtotal and total parotidectomy. $(2,3)$

This parotid surgery has several adverse effects, including facial palsy, sialocele or salivary fistula, sensory deficit, Frey's syndrome (FS), scars, and facial depression. $(4,5)$ Normally, the majority of facial palsy were temporary facial paresis. FS is common complication with affecting the quality of life. FS, also known as Gustatory Sweating syndrome, is characterized by gustatory sweating and gustatory flushing. (6) The incidence of FS has been reported in many articles with variable percentages. The overall average for FS is between $12.5 \%$ and $62 \%$ for subjective method and $86 \%$ to $98 \%$ when objective testing is performed with Minor's Starch-Iodine Test. $(7,8)$ Some patients report even having distressing symptoms. (9) Botulinum toxin A has been proved effective in the treatment of FS,7 however, its therapeutic modalities are rarely permanent in efficacy. (10) So, the trends of treatment have been build a barrier between skin and parotid gland. $(8,11)$

The facial depression in the periauricular and retromandibular areas were almost unavoidable with the removal of the parotid gland and tumors, which causing apparent asymmetry. The leads low aesthetic score, particularly in young people and those benign tumor patients. $(12,13)$ So, the areas need to be reconstructed with other tissues.

Some previous study have reported a variety of measures to prevent FS and the facial depression in the periauricular and retromandibular areas, such as a sternocleidomastoid, superficial muscular aponeurotic system, vascularized free flap, temporoparietal fascial flap, 
the free or vascularized dermal-fat graft, etc. (14-16) Those measures have its advantages and disadvantages. (11)

Acellular dermal matrix (ADM) can act as a biological scaffold for re-epithelialisation, neovascularisation, and infiltration of fibroblasts, but it does not produce an immune response. (17) It acts as an ideal alternative for tissue augmentation and barrier to preventing FS and facial depression after parotidectomy. $(18,19)$ The purpose of the present retrospective study was to investigate the complications and aesthetic effects of ADM after parotidectomy.

\section{METHODS}

Our study ruled the Declaration of Helsinki regarding ethics guidelines. The study protocol was approved by the '[removed for blind peer review]'. The latter waived written informed consent because of the retrospective nature of our study. Our ethics committee ('[removed for blind peer review]') approved the waiver of informed consent for this study.

All consecutive patients undergoing partial superficial or superficial or subtotal or total parotidectomy between January 2015 and December 2019 were included in this study. All patients underwent preoperative diagnosis by ultrasound or computed tomography (CT) scan. Patients were excluded from the study for the following reasons: (1) postoperative pathology suggests malignancy tumor; (2) previous history of radiation therapy or operated to the head and neck region; (3) incomplete medical records or follow-up. We collected all kinds of data including sex, age, type of tumor, type of parotidectomy, and tumor size, specimen volume, diabetes mellitus, hypertension, etc. The postoperative complication, drainage, aesthetic score outcome were analyzed. All patients were followed up for at least 6 months.

Parotidectomy was performed in the standard manner (Fig 1A, 1B). All parotid operations were performed by 1 of 2 experienced chief physician. The marginal mandibular of the facial nerve was first identified, and then a retrograde dissection of the nerve was performed. The acellular dermal matrix (ADM) used in our study was J1-ADM (Beijing Jayyalife Biological Technology Co. Ltd., Beijing, China). The ADM size was selected by the actual needs of operation. All patients were placed on prophylactic antibiotics for the duration their drain was in place.

Frey's syndrome (FS) and aesthetic score was evaluated by subjective methods. For the subjective FS evaluation, patients were queried about warming, flushing, or sweating over the preauricular regions during the follow-up period. For the subjective aesthetic score was achieved by asking patients to complete a questionnaire designed to assess their satisfaction with the resulting scar and upper cervical and retromandibular contour. Subjective aesthetic score was scaled from 1 to 10 (1 representing very dissatisfied; 10 representing very satisfied).(20)

The mean and variance were used as continuous variables and $\mathrm{n}(\%)$ were used as categorical variables. $\mathrm{T}$ test was used for continuous variables and Pearson Chi-square test or Fisher's exact test were used for categorical variables. $P<0.05$ was considered statistically significant. Statistical analysis was performed using SPSS 22.0 for Windows.

\section{RESULTS}

\section{Patient profiles}

Table 1 shows patient profiles before and after propensity score matching. A total of 175 patients with 33 patients in ADM group were enrolled in this study. Before propensity score-matching, the ADM group contained lower age, size of tumor, and volume of specimen, than those in the Control group. Moreover, the type of parotidectomy in the Control group were significantly difference than the type in the ADM group $(P$ $=0.008)$. As a result, the rate of male and hypertension in both groups was similar $(P=0.447, P=0.902)$. After propensity score-matching, both groups were well matched in all parameters. 30 patients each were allocated to the ADM group and the Control group. Pleomorphic adenomas and Warthin's tumors were the most frequent histological type in two groups. 25 patients (83\%) or 23 patients (77\%) underwent partial 
superficial parotidectomy, followed by superficial parotidectomy (4 patients in ADM group and 6 patients in Control group).

Complications

Overall complication rates are shown in Table 2. ADM group were associated with a total of 5 complications $(17 \%)$, whereas Control group were associated with a total of 13 complications (43\%) $(P=0.024)$.

Postoperative facial function was graded on the House-Brackmann scale in all patients. 3 patients (10\%) in the ADM group and 4 patients (13\%) in the Control group had facial palsy, with initially founded on the first day after operation. The postoperative facial palsy did not get statistical significance between two groups. All patients except the two Nerve Schwannoma patients fully regained normal facial function within 6 months. The two Nerve Schwannoma patients in either group developed permanent facial palsy with grade IV.

1 patient $(3 \%)$ in the ADM group and 6 patients (20\%) in the Control group developed a sialocele or salivary fistula. No significant difference was achieved between the two groups $(P=0.108)$.

$1(3 \%)$ patient in the ADM group and $9(30 \%)$ patients in the Control group experienced sweating over the preauricular regions 6 months after the operation. There was statistically significant difference between the two groups in subjective Frey syndrome (FS) $(P=0.015)$.

The most common complication was sensory deficit in both groups. Numbness in the infra-auricular region occurred in 4 patients (13\%) in the ADM group and in 7 patients $(23 \%)$ in the Control group $(P=0.317)$. 9 of the 11 patients with sensory deficit recovered sensation within 6 months after the operation.

None of the patients in the ADM group experienced complications at the donor site. No patient in either group had tumor recurrence and seromas during the follow-up period.

Postoperative drainage

The postoperative drainage in the ADM group was $59.7+26.0 \mathrm{~mL}$, whereas the postoperative drainage in the Control group was $110.0+78.7 \mathrm{~mL}$. Postoperative drainage was significantly decreased in the ADM group compared with the Control group (Fig. 2A) $(P=0.002)$. The postoperative drainage time in the ADM group was $3.6+0.8$ days, whereas the postoperative drainage time in the Control group was $4.7+1.7$ days. Postoperative drainage time was significantly lower in the ADM group compared with the Control group (Fig. 2B) $(P=0.001)$.

Expect the sialocele or salivary fistula patients, the postoperative drainage in the ADM group (29 patients) was $58.3+25.3 \mathrm{~mL}$, whereas the postoperative drainage in the Control group (24 patients) was $95.3+$ 72.6mL. Postoperative drainage was also significantly decreased in the ADM group compared with the Control group $(P=0.013)$. The postoperative drainage time in the ADM group was $3.6+0.8$ days, whereas the postoperative drainage time in the Control group was $4.5+1.6$ days. Postoperative drainage time was also significantly lower in the ADM group compared with the Control group $(P=0.010)$.

Subjective aesthetic score

Patients in the ADM group had a subjective aesthetic score of $6.1+1.7$ compared with $5.2+1.7$ in the Control group (Fig. 3). The subjective aesthetic score for patients in the ADM group was higher than those in the Control group $(P=0.040)$.

\section{DISCUSSION}

Facial nerve preservation is the most important problem in parotidectomy with benign tumors. However, quality of life after parotidectomy should not also be ignored. FS and facial aesthetic have the potential to profoundly impact quality of life after parotidectomy. So, the purpose of this study was to evaluate the complication, including FS, and aesthetic effects of insertion of ADM after parotidectomy with the method of propensity score matched analysis. 
One of the mechanisms of FS is caused by an aberrant regeneration between the postganglionic parasympathetic nerve fibers that innervate secretion of the parotid glands and the sympathetic nerve fibers that control the subcutaneous sweat glands and vessels after parotidectomy. (21) The reported that the overall incidence of FS following parotidectomy has been great difference. Most previous reported the subjective incidences of FS are between $10 \%$ and $40 \%$, and the objective incidences are up to $90 \%$.(22) One reason for this difference may lose to estimate the no clinic symptoms of gustatory sweating and and gustatory flushing. The other reason may the differences research methods. A reported that the incidence of FS in a prospective group was higher than in a retrospective one. The differences may be clarify that the prospective group was more aware of the possible incidence of FS and symptoms of FS may disappear with time in the retrospective group.

A variety of treatment have been reported over time with advantages and disadvantages. Botulinum toxin type A has become the first-line therapy. However, the durations effect may short from 12.1 months to 17.3 months. (23) Scholars have also reported that building a barrier in the surgical site after parotidectomy can help to prevent FS, including the sternocleidomastoid muscle flap, the temporoparietal fascia flap, the superficial musculoaponeurotic system flap(SMAS), the free or vascularized dermal fat graft, and ADM.

The SMAS layer is the superficial cervicofacial fascia. The SMAS flap is placed between the skin and the surgical site of the parotid region. The SMAS flap can effectively prevent the occurrence of FS following a parotidectomy in benign tumors. (24) The use of SMAS flap is limited in the malignant tumors or the obese patients, which is being resection or sometimes insufficient to cover the surgical site. Another limitation of the SMAS flap may more injure to the marginal mandibular branch or cervical branch of the facial nerve.

The free fat graft is also common barrier after parotidectomy. A reported that ADM and free fat grafts were an equal effective in preventing FS and other complications, including facial palsy, sialocele or salivary fistulas, and sensory deficit. (25) Nosan et al. (26) used free abdominal dermal-fat grafts to preserve the periauricular depression of 9 patients undergoing parotidectomy, and the results showed that the aesthetic scores was improving without significant complications. However, the shortcoming of grafts may be the need of a second surgical site with potential wound infection. The other shortcoming is the potentially unpredictable reabsorption rate without continuous efficiency. Some previous reported reabsorption rates were high with vary from $20 \%$ to $90 \%$. (11)

In recent years, ADM has been used in plastic surgery and parotidectomy. Hartzell et al. (27) reported ADM have a satisfactory effect and safe material for breast augmentation. In 2007, Chen et al. (28) reported ADM provided a satisfactory aesthetic result with a low incidence of FS in parotid haemangiomas. Govindaraj et al. (7) reported the use of ADM in the prevention of FS compare with control group for superficial parotidectomy. 64 patients were randomly equal assigned to two groups. The results demonstrated the subjective incidences of FS were 9.3\% in control group and 3.1\% in ADM group. The objective incidence of FS was $40 \%$ in control group and $0 \%$ in ADM group. However, the complication rate of $25 \%$ in ADM group was higher than $9 \%$ in control group. In 2008, Wei et al(29) reported similar conclusion in the incidence of FS after superficial and partial superficial parotidectomy. The subjective and objective incidences of FS were recorded in $61 \%$ and $23 \%$ from the control group and $2 \%$ and $2 \%$ from the ADM group. Furthermore, the complication of sialocele or salivary fistula in $17 \%$ from the control group was higher than that $2 \%$ from the ADM group. Luo et al. (19) reported the subjective incidences of FS in ADM group was 1 patient (3.4\%) lower than that 14 patients $(34.1 \%)$ in the control group for total parotidectomy. No cases of complication were observed in either group.

In our study, the subjective incidences of FS in ADM group were 1 patient (3\%) lower than that 9 patients $(30 \%)$ in the control group $(P=0.015)$. And the overall complication rate of $5(17 \%)$ in ADM group was lower than $13(43 \%)$ in control group $(P=0.024)$. The incidence of FS for both group in our study was resemble to the previous literature. $(7,19,28,29)$ The results of complication have been analogue to the conclusion of Wei et al(29), while difference from the conclusion of Govindaraj et al(7). The reason of difference may partly because the research methods were difference. Although our study has used retrospective study as same as the previous literature, we matched the baseline data according a propensity score matched analysis. 
We matched some important data including age, gender, type of tumor, size of tumor, volume of specimen, type of parotidectomy, hypertension and diabetes mellitus, which may reduce the bias of data and increase the persuasiveness of conclusions.

In our study, the complication of facial palsy and sensory deficit were no statistical significance. The incidence is related to the surgical technique, tumor size and location, individual feeling etc. The incidence rate of Sialocele or Salivary 1(3\%) in ADM group was lower than $6(20 \%)$ in control group $(P=0.108)$. However, postoperative drainage and time were significantly decreased or lower in the ADM group compared with the Control group (Fig. 2A and 2B). In the present study, Although the incidence rate of Sialocele or Salivary was no statistical significance, ADM patients experienced a lower rate of postoperative drainage and time when compared with control group. This difference of Sialocele or Salivary and postoperative drainage were thought to be related to the use of the barrier of ADM. Some previous study has shown similar conclusion. Ye et al. (29) reported one salivary fistula was noted in the ADM group, while 18 cases developed in the control group $(P=0.002)$. We consider that the ADM should completely cover the exposed parotid and facial nerve surface. ADM graft may replace fascia to reduce secretion and promptly eliminate the dead space. When the postoperative drainage is less than $10 \mathrm{ml}$ drainage tube can be removed, and a proper pressure dressing used for about 3-5 days. So, the ADM graft may promote the quality of life after parotidectomy.

The aesthetic score after parotidectomy should also be important considerations. In our study, the subjective aesthetic score for patients in the ADM group was higher than those in the Control group $(P=0.040)$. In 2012, a study reported the use of ADM may reduce postoperative facial contour deformities following total parotidectomy. (19) Ciuman et al(30) also reported that the extent of parotidectomy was related to aesthetic score. In 2019, Kim et al. (9) shown insertion of ADM after parotidectomy, including partial, superficial, and total parotidectomy, may improve aesthetic score. The results also demonstrated the aesthetic score was related to gender and complications. Interestingly, the results showed that women were more satisfied than men. We consider the subjective aesthetic score may no difference in both group before the study. Our research results indicated the factor of subjective aesthetic score are manifold. We guess the factor is related to the age, gender, incision, size of tumor, sample volume, complications, individual feelings etc. There are need further study.

Our study was limited by the small sample, relatively short follow-up time. The FS and aesthetic score were evaluated by subjective methods without objectively assess. The reason is the FS and aesthetic score are higher related to the subjective evaluation than the objective evaluation. So, even if the starch iodine test is positive, or the aesthetic score is low, if a patient suffers no symptoms or satisfied then no intervention is required.

In conclusion, the present clinical study suggests that ADM graft are effective in preventing FS and improving aesthetic score after parotidectomy. More RCTs are needed to confirm

this conclusion and prove the influencing factors of aesthetic score.

\section{Data availability statement}

The datasets generated and analyzed during the current study are available from the corresponding author on reasonable request.

Conflict of interest statement: The authors declare no potential conflicts of interest.

\section{References}

1. Skolnik EM, Friedman M, Becker S, Sisson GA, Keyes GR. Tumors of the major salivary glands. 1977;87(6):843-61.

2. Stennert E, Guntinas-Lichius O, Klussmann JP, Arnold G. Histopathology of Pleomorphic Adenoma in the Parotid Gland: A Prospective Unselected Series of 100 Cases. 2001;111(12):2195-200. 
3. McMullen CP, Smith RV, Ow TJ, Tassler A, Schiff BA. Minimal Margin Extracapsular Dissection:A Viable Alternative Technique for Benign Parotid Lesions? 2016;125(11):912-7.

4. Durgut O, Basut O, Demir UL, Özmen ÖA, Kasapoglu F, Coskun H. Association between skin flap thickness and Frey's syndrome in parotid surgery. 2013;35(12):1781-6.

5. Bulut OC, Plinkert P, Federspil PAJEAoO-r-l. Modified facelift incision for partial parotidectomy versus bayonet-shaped incision: a comparison using visual analog scale. 2016;273(10):3269-75.

6. Barberá R, Castillo F, D’Oleo C, Benítez S, Cobeta I. Superficial musculoaponeurotic system flap in partial parotidectomy and clinical and subclinical Frey's syndrome. Cosmesis and quality of life. 2014;36(1):130-6.

7. Govindaraj S, Cohen M, Genden EM, Costantino P, Urken MLJL. The Use of Acellular Dermis in the Prevention of Frey's Syndrome. 2001;111(11):1993-8.

8. Dulguerov P, Quinodoz D, Cosendai G, Piletta P, Marchal F, Lehmann W. Prevention of Frey Syndrome During Parotidectomy. Archives of Otolaryngology-Head \& Neck Surgery. 1999;125(8):833-9.

9. Kim DY, An SH, Woo J, Baek M, Kang IGJBJoO, Surgery M. Cosmetic outcome of implantation of cross-linked human acellular dermal matrix after parotidectomy. 2019;57(4):301-5.

10. Aradcohen A, Blitzer AJO-H, Surgery N. Botulinum toxin treatment for symptomatic Frey's syndrome. 2000;122(2):237-40.

11. Chandarana S, Fung K, Franklin JH, Kotylak T, Matic DB, Yoo JJH, et al. Effect of autologous platelet adhesives on dermal fat graft resorption following reconstruction of a superficial parotidectomy defect: A double-blinded prospective trial. 2009;31(4):521-30.

12. Bianchi B, Ferri A, Ferrari S, Copelli C, Sesenna EJJoO, Surgery M. Improving Esthetic Results in Benign Parotid Surgery: Statistical Evaluation of Facelift Approach, Sternocleidomastoid Flap, and Superficial Musculoaponeurotic System Flap Application. 2011;69(4):1235-41.

13. Kerawala CJ, Mcaloney N, Stassen LFAJBJoO, Surgery M. Prospective randomised trial of the benefits of a sternocleidomastoid flap after superficial parotidectomy. 2002;40(6):468-72.

14. Sachsman SM, Rice DHJE, nose, journal t. Use of AlloDerm implant to improve cosmesis after parotidectomy. 2007;86(8):512-3.

15. Yoo YM, Lee JS, Park MC, Kim C, Seo SJ, Lee IJJJoCS. Dermofat Graft After Superficial Parotidectomy Via a Modified Face-Lift Incision to Prevent Frey Syndrome and Depressed Deformity. 2011;22(3):1021-3.

16. Curry J, Fisher KW, Heffelfinger R, Rosen M, Keane WM, Pribitkin EAJL. Superficial Musculoaponeurotic System Elevation and Fat Graft Reconstruction After Superficial Parotidectomy. 2008;118(2):210-5.

17. Lee JH, Kim HG, Lee WJJB. Characterization and tissue incorporation of cross-linked human acellular dermal matrix. 2015;44:195-205.

18. Tamplen M, Knott PD, Fritz MA, Seth RJFPSCoNA. Controversies in Parotid Defect Reconstruction. 2016;24(3):235-43.

19. Luo W, Zheng X, Chen L, Jing W, Tang W, Long J, et al. The use of human acellular dermal matrix in the prevention of infra-auricular depressed deformities and Frey's syndrome following total parotidectomy. $2012 ; 114(2)$.

20. Liu H, Li Y, Dai XJOSOMOPOR, Endodontology. Modified face-lift approach combined with a superficially anterior and superior-based sternocleidomastoid muscle flap in total parotidectomy. 2012;113(5):593-9.

21. Dulguerov P, Marchal F, Gysin CJL. Frey syndrome before Frey: the correct history. 1999;109(9):1471-3.

22. De Bree R, Der Waal IV, Leemans CRJH, Sciences N-jfT, Head SoT, Neck. Management of frey syndrome. 2007;29(8):773-8. 
23. Laskawi R, Drobik C, Schonebeck CJL. Up-to-date Report of Botulinum Toxin Type A Treatment in Patients With Gustatory Sweating (Frey's Syndrome). 1998;108(3):381-4.

24. Meningaud J, Bertolus C, Bertrand JJJoC-mS. Parotidectomy: Assessment of a surgical technique including facelift incision and SMAS advancement. 2006;34(1):34-7.

25. Wang S, Li L, Chen J, Li X, Yin J, Liu K, et al. Effects of free fat grafting on the prevention of Frey's syndrome and facial depression after parotidectomy: A prospective randomized trial. 2016;126(4):815-9.

26. Nosan DK, Ochi JW, Davidson TMJO-H, Surgery N. Preservation of facial contour during parotidectomy. 1991;104(3):293-8.

27. Hartzell TL, Taghinia AH, Chang J, Lin SJ, Slavin SAJP, Surgery R. The use of human acellular dermal matrix for the correction of secondary deformities after breast augmentation: results and costs. 2010;126(5):1711-20.

28. Chen W, Li J, Yang Z, Yongjie W, Zhiquan W, Wang YJJoPR, et al. SMAS fold flap and ADM repair of the parotid bed following removal of parotid haemangiomas via pre- and retroauricular incisions to improve cosmetic outcome and prevent Frey's syndrome. 2008;61(8):894-9.

29. Ye W, Zhu HG, Zheng JW, Wang XD, Zhao W, Zhong LP, et al. Use of allogenic acellular dermal matrix in prevention of Frey's syndrome after parotidectomy. 2008;46(8):649-52.

30. Ciuman RR, Oels W, Jaussi R, Dost P. Outcome, general, and symptom-specific quality of life after various types of parotid resection. 2012;122(6):1254-61.

\section{Figure legends}

Fig 1. (A) Partial resection of superficial lobe and tumor. (B) acellular dermal matrix implantation.

Fig 2. (A)Comparison of postoperative drainage between two groups. (B) Comparison of postoperative drainage time between two groups.

Fig 3. Comparison of aesthetic scores between two groups. 


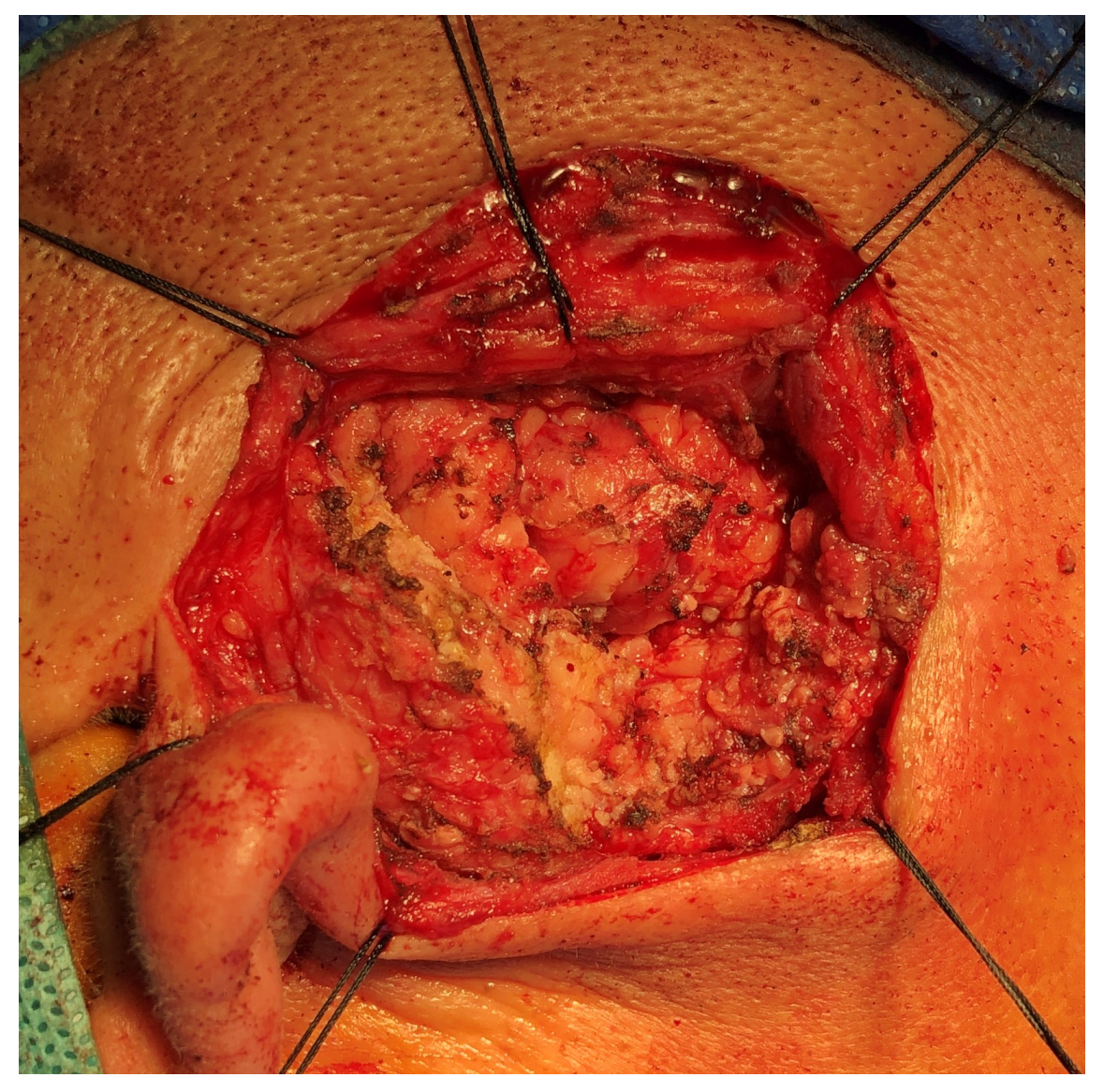



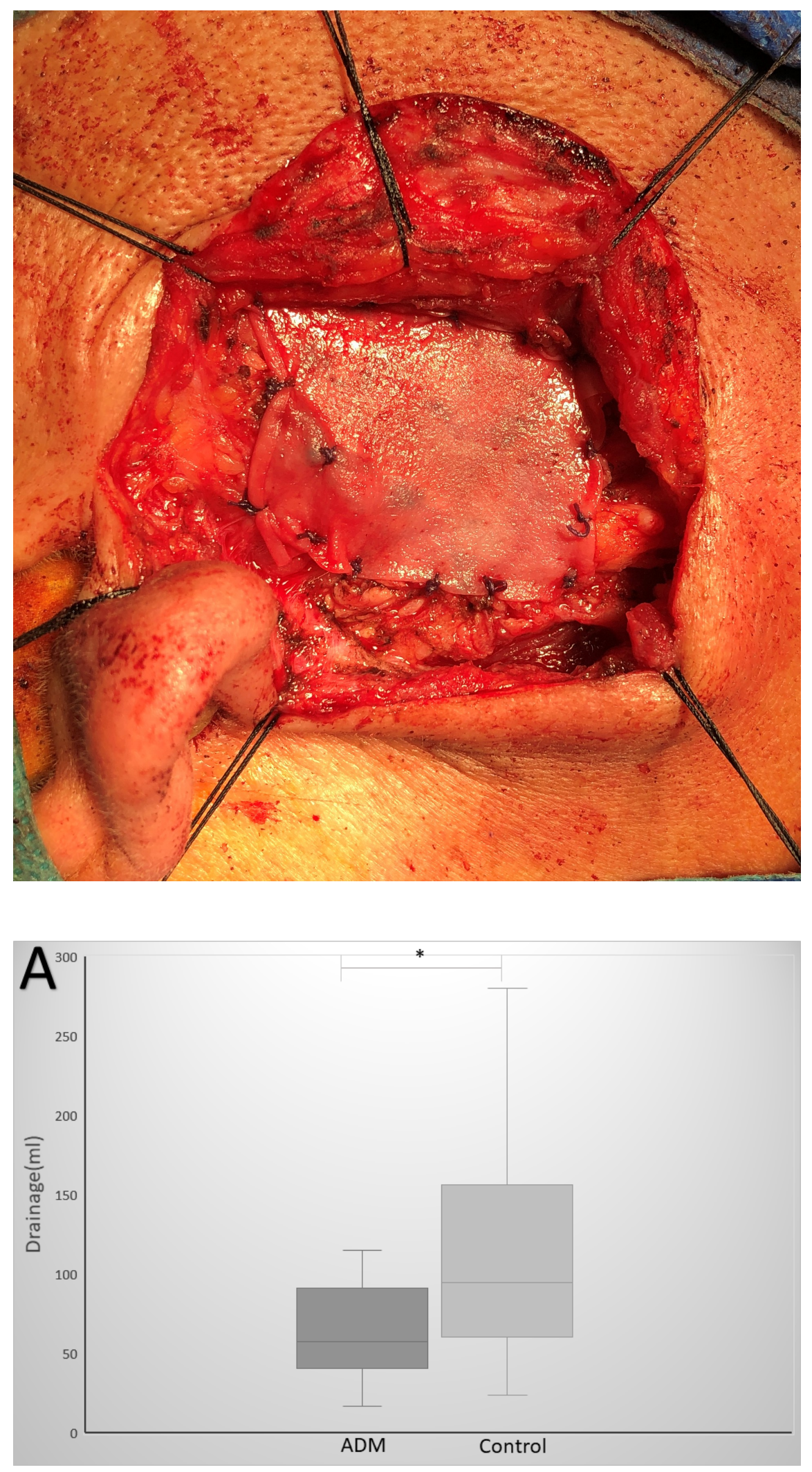

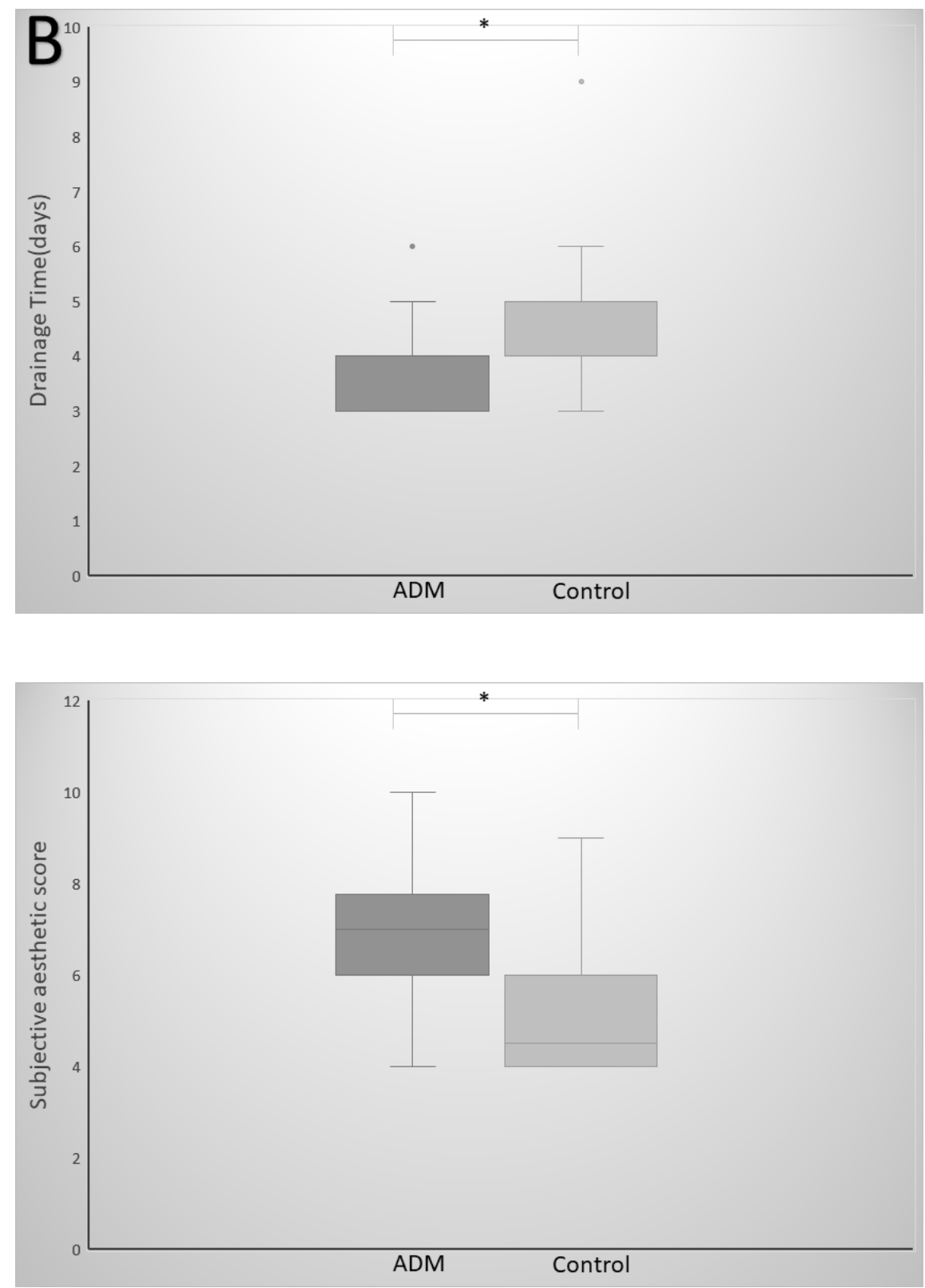

\section{Hosted file}

Table 1.pdf available at https://authorea.com/users/363521/articles/484260-effects-ofacellular-dermal-matrix-on-the-prevention-of-complications-and-aesthetic-outcome-afterparotidectomy

\section{Hosted file}

Table 2.pdf available at https://authorea.com/users/363521/articles/484260-effects-ofacellular-dermal-matrix-on-the-prevention-of-complications-and-aesthetic-outcome-afterparotidectomy 